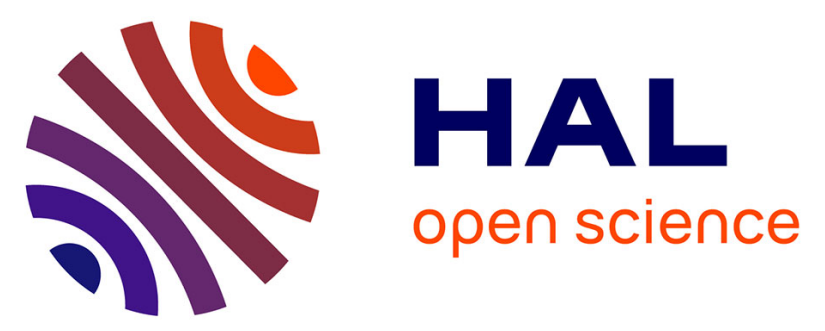

\title{
Chemical Tuning of Magnetic Exchange Couplings Using Broken-Symmetry Density Functional Theory
}

\author{
Grégoire David, Frank Wennmohs, Frank Neese, Nicolas Ferré
}

\section{To cite this version:}

Grégoire David, Frank Wennmohs, Frank Neese, Nicolas Ferré. Chemical Tuning of Magnetic Exchange Couplings Using Broken-Symmetry Density Functional Theory. Inorganic Chemistry, 2018, 57 (20), pp.12769-12776. 10.1021/acs.inorgchem.8b01970 . hal-02053142

\section{HAL Id: hal-02053142 \\ https://hal-amu.archives-ouvertes.fr/hal-02053142}

Submitted on 1 Mar 2019

HAL is a multi-disciplinary open access archive for the deposit and dissemination of scientific research documents, whether they are published or not. The documents may come from teaching and research institutions in France or abroad, or from public or private research centers.
L'archive ouverte pluridisciplinaire HAL, est destinée au dépôt et à la diffusion de documents scientifiques de niveau recherche, publiés ou non, émanant des établissements d'enseignement et de recherche français ou étrangers, des laboratoires publics ou privés.

\section{(c)(1)}

Distributed under a Creative Commons Attribution| 4.0 International License 


\title{
Chemical tuning of magnetic exchange couplings using broken-symmetry density-functional theory
}

\author{
Grégoire David, ${ }^{\dagger}$ Frank Wennmohs, ${ }^{\ddagger}$ Frank Neese ${ }^{\ddagger}$ and Nicolas Ferré*,† \\ $\dagger$ †ix-Marseille Univ, CNRS, ICR, Marseille, France \\ $\ddagger$ Max-Planck-Institüt für Kohlenforschung, Kaiser-Wilhelm-Platz 1, Mülheim an der Ruhr, \\ 45470 North Rhine-Westphalia, Germany \\ E-mail: nicolas.ferre@univ-amu.fr
}

\begin{abstract}
Using broken-symmetry Kohn-Sham Density-Functional Theory calculations, it is demonstrated that the ferromagnetic or anti-ferromagnetic character of two prototypical binuclear copper complexes can be modified, both in the sign and in magnitude, by means of chemical substitutions operated on the bridges connecting the two magnetic centers. The level of detail provided by the magnetic exchange decomposition in terms of direct exchange, kinetic exchange and core polarization puts forward the relative importance of the different bridges. At variance with the principal bridge for which chemical substitutions modify both the direct and the kinetic exchange contributions, modifications of the secondary bridge only affect the magnitude of the antiferromagnetic kinetic exchange mechanism, ultimately allowing for a direct control of the magnetic character of the modified compound.
\end{abstract}




\section{Introduction}

Magnetic exchange coupling $J$ is one of the most important quantum interactions between two (or more) unpaired electrons. ${ }^{1}$ In the case of a finite-size systems, this interaction is important for designing molecular compounds with controlled magnetic properties. When $J$ is non-zero in a molecule, it favors either the parallel alignment of the magnetic electron spin angular momenta (ferromagnetic interaction) or the anti-parallel one (antiferromagnetic interaction), eventually driving the magnetic properties of the system. Accordingly, the determination of $J$, either experimentally ${ }^{2-4}$ or by means of quantum-chemistry calculations, ${ }^{5-20}$ is of tremendous importance for understanding the behavior of magnetic open-shell molecular systems.

Different physical mechanisms may compete and determine the sign and intensity of $J$. In the case of two spin-1/2 magnetic centers, the most important ones are the direct exchange and the kinetic exchange between the magnetic electrons, and the polarization of the nonmagnetic electrons. While high level quantum-mechanical methods can accurately evaluate the importance of these mechanisms, eg by selectively considering classes of excitations in configuration interaction calculations, ${ }^{21}$ their computational cost limits their applicability to small molecules. On the other hand, the Kohn-Sham density-functional theory combined with the so-called broken-symmetry approach (BS-KSDFT) is a popular and effective method which often provides semi-quantitative evaluations of $J^{7,15,22}$ By means of selective freezing of a subset of orbitals, some of the present authors recently introduced a decomposition scheme ${ }^{23-25}$ which has been essentially applied to centro-symmetric molecules until now. ${ }^{26}$

Thanks to the recent implementation of this method in the Orca package, ${ }^{27}$ the $J$ decomposition is now available for molecules belonging to various symmetry groups, hence expanding its range of application to systems where the magnetic centers are bridged by chemically different moieties, eg $\pi$-conjugated or not, with electron-donating or electron-withdrawing substituents, etc. Hereafter, we report $J$ decompositions for various non-centrosymmetric copper dinuclear compounds. All of them feature oxygen- or carboxylic-bridged cores, with 
different chemical substitutions. In all, it is demonstrated that the deep understanding of the physical contributions to $J$ can be used to in-silico design molecular compounds with selected magnetic properties.

\section{Molecular models}

Among all the possible, available, non-centrosymmetric copper dinuclear complexes that exhibit significant magnetic exchange couplings, we have selected $\left[\{\mathrm{Cu}(\mathrm{dmen})\}_{2}(\mu-\mathrm{OMe})\left\{\mu-\left[\mathrm{O}_{2} \mathrm{C}\left(\eta^{5}-\mathrm{C}_{5} \mathrm{H}_{5}\right)\right.\right.\right.$ (hereafter identified as PATFIA) and its closely related analogue $\left[\{\mathrm{Cu}(\text { tmen })\}_{2}(\mu-\mathrm{OH})\left(\mu-\mathrm{O}_{2} \mathrm{ClO}_{2}\right)\left\{\mu-\left[\mathrm{O}_{2}\right.\right.\right.$ (hereafter identified as PATFOG). Both PATFIA and PATFOG, whose synthesis have been reported in $2005,{ }^{28}$ feature two different bridges (Figure 1). Moreover, inspection of the corresponding experimental crystallographic structures reveal a close contact with 1 or 2 perchlorate counterions. From experiment, the two copper ions in PATFIA have been shown
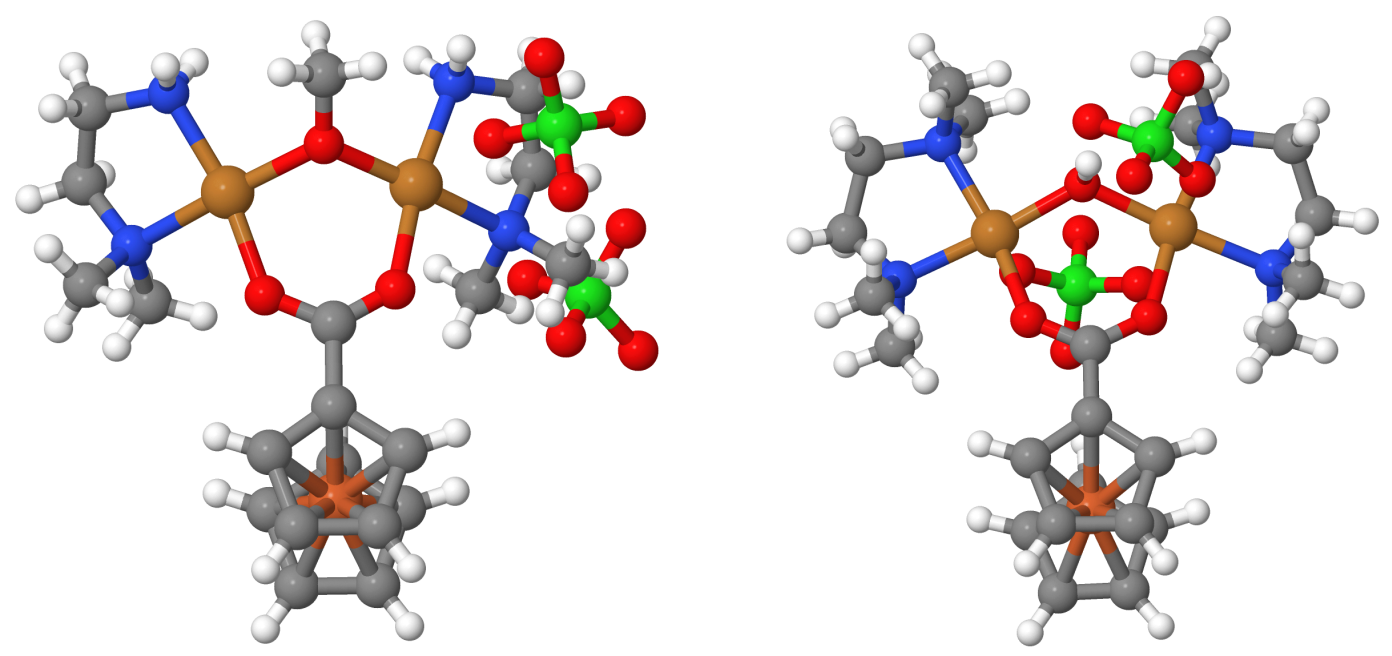

Figure 1: Molecular structures of PATFIA (left) and PATFOG (right).

to be weakly antiferromagnetically coupled $\left(J=-5.5 \mathrm{~cm}^{-1}\right)$, while PATFOG shows ferromagnetic coupling $\left(J=14.7 \mathrm{~cm}^{-1}\right)$, both $J$ values being defined through the $\mathcal{H}=-2 J \mathcal{S}_{A} \cdot \mathcal{S}_{B}$ spin Hamiltonian. In the same article ${ }^{28}$ quantum chemical calculations have been used to understand the origin of the different couplings in PATFIA and PATFOG. The out-of-plane angle of the hydroxo-based bridge side-chain is found to be the principal structural param- 
eter controlling the ferro- vs antiferromagnetic character of PATFOG vs PATFIA. Since the publication of this article, PATFIA has been repeatedly used as a benchmark molecule for evaluating the accuracy of various quantum-mechanical methods. The corresponding $J$ values are reported in Table 1, together with the model details. In almost every reported studies, the perchlorate counterions have been ignored, while the ferrocene moiety is simply replaced by a methyl group or a hydrogen atom. Looking at Table 1 entries, it is quite obvious that the correct evaluation of PATFIA $J$ value depends on a large number of parameters: the DFT xc-functional, the geometry, the basis set, the method (BS vs other ones) etc. While some models perform wrong, even qualitatively (ferromagnetic instead of anti-ferromagnetic), some other ones overshoot the coupling by two orders of magnitude. On the other hand, besides the original calculations performed on PATFOG, ${ }^{28}$ we are aware of only a very recent theoretical study dealing with this system, ${ }^{36}$ for which a large set of DFT functionals have been tested, with $J$ values ranging from $-3.6 \mathrm{~cm}^{-1}$ to $59.9 \mathrm{~cm}^{-1}$. Notice that all but one DFT functional predict PATFOG as ferromagnetic.

In the present work, the starting structures have been retrieved from the Cambridge Structural Database. ${ }^{37}$ Comparing PATFIA and PATFOG structures, several differences show up. First, one of the bridges is hydroxo in PATFOG, while it is methoxo in PATFIA. In the case of PATFOG, the hydroxo moiety is oriented in such a way it can form a hydrogen bond with one perchlorate molecule. This orientation is thought to govern the magnetic coupling character of these systems. Second, external ligands are dimethylethylenediamine in PATFIA, while they are tetramethylethylenediamine in PATFOG, hence feature two more methyl groups. Accordingly, it would be interesting to assess the influence on $J$ of these two extra chemical groups. Third, the perchlorate anions are distributed differently in PATFIA and PATFOG, the latter featuring a hydrogen bond with the hydroxo bridge. While these anions are usually removed from the theoretical models, the original work from López et al ${ }^{28}$ insisted on the structural importance of perchlorate counterions, constraining the orientation of the hydroxo bridge, ultimately modifying PATFOG magnetic properties. 
Table 1: Experimental (in bold) $J$ value complemented with some examples of PATFIA models reported in the literature, with the corresponding level of theory, the type of geometry (either experimental (exp) or optimized (opt), the inclusion of ferrocene (if not, the nature of the substituted fragment) and the corresponding $J$ values in $\mathrm{cm}^{-1}$. BS: Broken-Symmetry, TD: Time-Dependent, REKS: Restricted-Ensemble Kohn-Sham, $\mathrm{SF}_{c}$ : collinear Spin-Flip, $\mathrm{SF}_{n c}$ :non-collinear Spin-Flip, CV: Constricted Variational. DFT xc-functional is given in parenthesis, while other model details and basis sets are available in the above references.

\begin{tabular}{|c|c|c|c|c|}
\hline Model & Level of theory & Geometry & Ferrocene & $J$ \\
\hline Ref 28 & $\mathcal{H}=-2 J \mathcal{S}_{A} \cdot \mathcal{S}_{B}$ & $\exp$ & yes & -5.5 \\
\hline Ref 28 & CASPT2 & opt & no, $\mathrm{H}$ & -9 \\
\hline Ref 28 & BS(B3LYP) & $\exp$ & no, $\mathrm{H}$ & -31 \\
\hline Ref 29 & BS(BH\&HLYP) & $\exp$ & no, $\mathrm{H}$ & +5 \\
\hline Ref 29 & REKS(B3LYP) & $\exp$ & no, $\mathrm{H}$ & +70 \\
\hline Ref 29 & REKS(BH\&HLYP) & $\exp$ & no, $\mathrm{H}$ & +16 \\
\hline Ref 30 & BS(M06) & $\exp$ & no, $\mathrm{H}$ & -8 \\
\hline Ref 30 & $\mathrm{BS}(\mathrm{M} 06-2 \mathrm{X})$ & $\exp$ & no, $\mathrm{H}$ & -9 \\
\hline Ref 30 & $\mathrm{BS}(\mathrm{M} 06-\mathrm{HF})$ & $\exp$ & no, $\mathrm{H}$ & -19 \\
\hline Ref 30 & $\mathrm{BS}(\mathrm{PBE} 0)$ & $\exp$ & no, $\mathrm{H}$ & -18 \\
\hline Ref 31 & $\mathrm{BS}(\mathrm{HSE})$ & $\exp$ & no, $\mathrm{H}$ & -19 \\
\hline Ref 31 & $\mathrm{BS}(\mathrm{LC}-\omega \mathrm{PBE})$ & $\exp$ & no, $\mathrm{H}$ & -19 \\
\hline Ref 32 & $\mathrm{SF}_{c^{-}} \mathrm{TD}(\mathrm{B} 3 \mathrm{LYP})$ & $\exp$ & no, $\mathrm{H}$ & -200 \\
\hline Ref 32 & $\mathrm{SF}_{c}-\mathrm{TD}(\mathrm{B} 3 \mathrm{LYP})$ & $\exp$ & yes & -210 \\
\hline Ref 32 & $\mathrm{SF}_{c}-\mathrm{TD}(\mathrm{B} 3 \mathrm{LYP} 40)$ & $\exp$ & no, $\mathrm{H}$ & -200 \\
\hline Ref 32 & $\mathrm{SF}_{c}-\mathrm{TD}(\mathrm{B} 3 \mathrm{LYP} 40)$ & $\exp$ & yes & -49 \\
\hline Ref 32 & $\mathrm{SF}_{c^{-}} \mathrm{TD}(\mathrm{BMK})$ & $\exp$ & no, $\mathrm{H}$ & -36 \\
\hline Ref 32 & $\mathrm{SF}_{c^{-}} \mathrm{TD}(\mathrm{BMK})$ & $\exp$ & yes & -43 \\
\hline Ref 32 & $\mathrm{SF}_{c^{-}} \mathrm{TD}(\mathrm{M} 06-2 \mathrm{X})$ & $\exp$ & no, $\mathrm{H}$ & -11 \\
\hline Ref 32 & $\mathrm{SF}_{c}-\mathrm{TD}(\mathrm{M} 06-2 \mathrm{X})$ & $\exp$ & yes & -12 \\
\hline Ref 33 & $\mathrm{SF}_{c}-\mathrm{CV}(\mathrm{B} 3 \mathrm{LYP})$ & $\exp$ & no, $\mathrm{H}$ & -43 \\
\hline Ref 33 & $\mathrm{SF}_{c}-\mathrm{CV}(\mathrm{BH} \& \mathrm{HLYP})$ & $\exp$ & no, $\mathrm{H}$ & -10 \\
\hline Ref 34 & $\mathrm{BS}(\mathrm{LDA}+\mathrm{U})$ & $\exp$ & no, $\mathrm{H}$ & +18 \\
\hline Ref 34 & $\mathrm{BS}(\mathrm{GGA}+\mathrm{U})$ & $\exp$ & no, $\mathrm{H}$ & -36 \\
\hline Ref 16 & BS(STPSS) & $\exp$ & no, $\mathrm{H}$ & -307 \\
\hline Ref 16 & BS(PBETPSS) & $\exp$ & no, $\mathrm{H}$ & -246 \\
\hline Ref 16 & BS(TPSSTPSS) & $\exp$ & no, $\mathrm{H}$ & -188 \\
\hline Ref 35 & $\mathrm{SF}_{c}-\mathrm{TD}(\mathrm{B} 5050 \mathrm{LYP})$ & $\exp$ & no, $\mathrm{H}$ & -56 \\
\hline Ref 35 & $\mathrm{SF}_{n c}-\mathrm{TD}(\mathrm{B} 5050 \mathrm{LYP})$ & $\exp$ & no, $\mathrm{H}$ & +2 \\
\hline Ref 35 & $\mathrm{SF}_{n c}-\mathrm{TD}(\mathrm{B} 5050 \mathrm{LYP})$ & opt & no, $\mathrm{H}$ & -25 \\
\hline Ref 35 & $\mathrm{SF}_{c}-\mathrm{TD}(\mathrm{B} 5050 \mathrm{LYP})$ & $\exp$ & yes & -60 \\
\hline
\end{tabular}

Together with the evaluation of the ferrocene effect on $J$ (which has been recently confirmed to be innocent ${ }^{35}$ ), we intend to study how these chemical differences and substitutions 
influence the different physical contributions to the magnetic exchange couplings in PATFIA and PATFOG, thanks to the BS-KSDFT-based decomposition scheme, as presented in the next section.

\section{Methods}

Our $J$ decomposition scheme is based on successive BS-KSDFT calculations, optimizing either restricted open-shell (RO) or unrestricted (U) Kohn-Sham wavefunctions under the constraint that a subset of orbitals (either the magnetic or the non-magnetic ones) are kept frozen. In the case of two magnetic centers $A$ and $B$, each of them carrying a single unpaired electron, we make use of the following effective spin-Hamiltonian:

$$
\mathcal{H}=-2 J \mathcal{S}_{A} \cdot \mathcal{S}_{B}
$$

for which $J$ can be decomposed using the Heisenberg-Dirac-van Vleck model as:

$$
J=J_{0}+\Delta J_{k e}+\Delta J_{c p}
$$

ie, $J$ is the sum of three important contributions: the direct exchange, the kinetic exchange and the so-called core polarization of the non-magnetic orbitals. The first step of the procedure consists in the calculation of the magnetic orbitals. Using a high-spin state (a triplet, in the case of two spin-1/2 sites) RO solutions ( $T, R O)$, both singly-occupied molecular orbitals are localized, using one of the available localizations schemes in Orca. ${ }^{27}$ Such schemes include the popular Pipek-Mezey ${ }^{38}$ and Boys ${ }^{39}$ localization schemes as well as the more recently developed method of intrinsic atomic orbitals. All schemes are combined with a robust and efficient search algorithm that is designed to avoid local minima of the localization functional to the largest extent.

The remaining steps are hereafter summarized: 
1. Flip the spin of one magnetic orbital to produce the broken-symmetry $M_{s}=0$ wavefunction (BS,RO), without optimizing any orbital, and evaluate the direct exchange contribution $J_{0}$ :

$$
J_{0}=E_{B S, R O}-E_{T, R O}
$$

2. Relax the magnetic orbitals in the $M_{s}=0$ wavefunction, in the field of the frozen non-magnetic orbitals, allowing the magnetic orbitals to delocalize from one center to the other (BS,UFC):

$$
\Delta J_{k e}=\frac{1}{\left\langle S^{2}\right\rangle_{T, R O}-\left\langle S^{2}\right\rangle_{B S, U F C}}\left(E_{B S, U F C}-E_{T, R O}\right)-J_{0}
$$

3. Relax the core orbitals in both high-spin $\left(M_{s}=1\right)$ and broken-symmetry $M_{s}=0$ wavefunctions (respectively T,UFM and BS,UFM):

$$
\Delta J_{c p}=\frac{\left(E_{B S, U F M}-E_{T, U F M}\right)}{2-\left(\left\langle S^{2}\right\rangle_{B S, U F C}+\left\langle S^{2}\right\rangle_{B S, U F M}\right) / 2+\left\langle S^{2}\right\rangle_{B S, U F C}\left(\left\langle S^{2}\right\rangle_{B S, U F M}-\left\langle S^{2}\right\rangle_{B S, U F C}\right) / 2}-J_{0}-\Delta J_{k e}
$$

4. Total magnetic coupling is obtained with the complete relaxation of orbitals in both high-spin $\left(M_{s}=1\right)$ and broken-symmetry $M_{s}=0$ wavefunctions (respectively $\mathrm{T}, \mathrm{U}$ and $\mathrm{BS}, \mathrm{U})$ :

$$
J_{T o t}=\frac{\left(E_{B S, U}-E_{T, U}\right)}{2-\left(\left\langle S^{2}\right\rangle_{B S, U F C}+\left\langle S^{2}\right\rangle_{B S, U}\right) / 2+\left\langle S^{2}\right\rangle_{B S, U F C}\left(\left\langle S^{2}\right\rangle_{B S, U}-\left\langle S^{2}\right\rangle_{B S, U F C}\right) / 2}
$$

Freezing orbitals is achieved thanks to the Local Self-Consistent-Field method, ${ }^{40}$ in which the relaxed orbitals are variationally optimized under the constraint of being orthogonal to the frozen ones.

Because the various orbital relaxations are performed in the unrestricted formalism, the resulting Kohn-Sham wavefunctions are spin-contaminated, ie characterized by a $\left\langle\mathcal{S}^{2}\right\rangle$ 
value different from 2 (high-spin) or 0 (low-spin). Consequently, we make use of spindecontamination schemes in all the three steps. While the Yamaguchi procedure can be applied safely to the direct and kinetic exchange contributions, it is no longer the case for core polarization, as demonstrated elsewhere. ${ }^{25}$

The selection of a xc functional is known to be very delicate in BS-KSDFT $J$ calculations. Even the physical interpretation of the contributions to $J$ depends on the chosen xc

functional. ${ }^{41}$ In this work, we have followed the very recent advice by Illas and coworkers, ${ }^{36}$ recommending to use the very common B3LYP functional for obtaining qualitative (ferromagnetic vs anti-ferromagnetic) to semi-quantitative (a given order of magnitude) results. Our choice is further motivated by the main goal of the current study, in which we will compare chemically-related compounds which differ only by one or two substitutions. Regarding the basis set, we have also followed recommendations by others, selecting a fairly large basis set for copper atoms (def2-QZVPP) while keeping reasonable ones for the other atoms (def2-SVP). The quality of the basis set and the possible influence of relativistic effects have been considered and are reported in Supporting Information.

Our results are discussed on the basis of the Mulliken atomic spin populations, complemented with spin density maps calculated at different stages of the decomposition scheme.

\section{Results}

We have first tested the influence of the ferrocene and solvent molecules on the $J$ decomposition results. Similarly to what has been reported by other (references in Table 1), our results available as Supporting Information indicate we can safely ignore them while retaining a good description of PATFIA and PATFOG magnetic properties. 


\section{Effect of chemical substitutions}

PATFIA and PATFOG feature two different bridges which chemical nature may modify the coupling between their two copper-centered magnetic electrons. One of them is either hydroxo or methoxo, with only one oxygen atom. Oxygen-bounded moieties will be called R1 hereafter (Figure 2). As already pointed in reference 28, their different orientations are

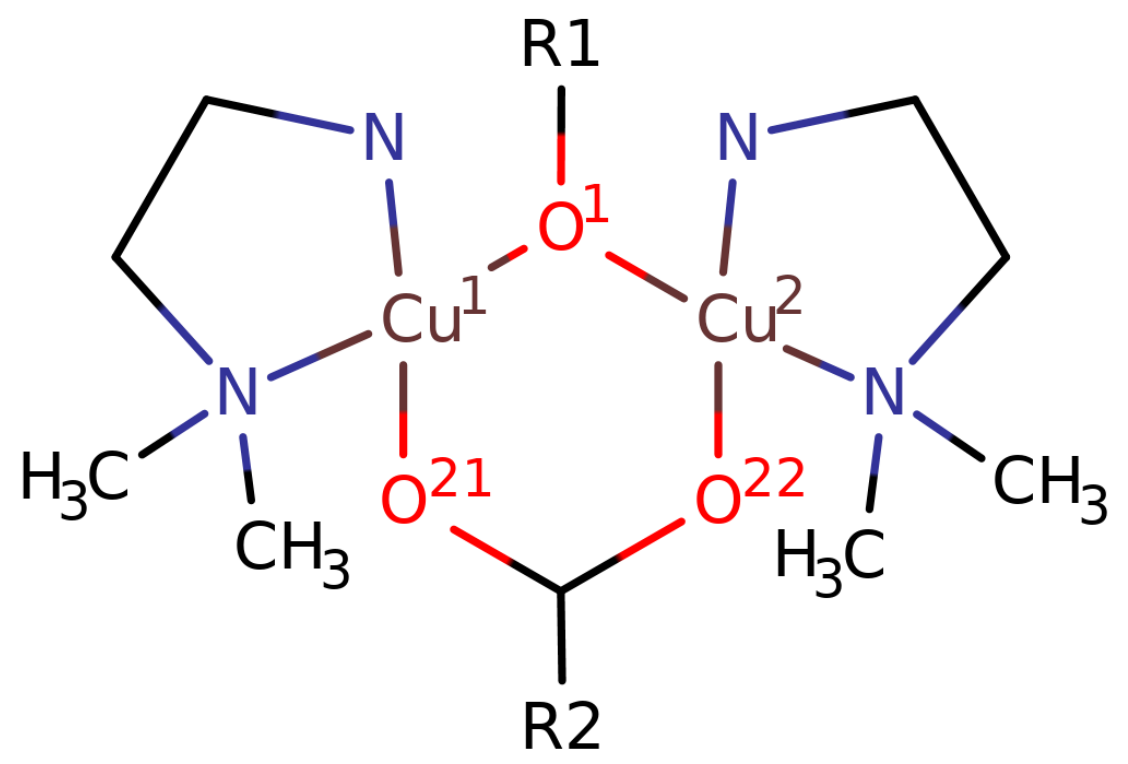

Figure 2: Molecular structures of modified PATFIA models. R1 and R2 are the sites of substitution. H atoms are omitted for clarity.

responsible for PATFIA and PATFOG different magnetic characters. The other bridge is carboxylato, complemented with ferrocene. Carbon-bounded chemical group will be called R2 hereafter. In the following, we report computational experiments of $J$ decomposition for PATFIA and modified PATFIA models (no perchlorate) in which R1 and R2 can be hydrogen $(-\mathrm{H})$, methyl $\left(-\mathrm{CH}_{3}\right)$, trifluoromethyl $\left(-\mathrm{CF}_{3}\right)$, tertiobutyl $\left(-\mathrm{C}\left(\mathrm{CH}_{3}\right)_{3}\right)$, trimethylformate $\left(-\mathrm{C}\left(\mathrm{OCH}_{3}\right)_{3}\right)$, triaminomethyl $-\mathrm{C}\left(\mathrm{NH}_{2}\right)_{3}$ or $\mathrm{N}$-dimethylphosphine $-\mathrm{P}\left(\mathrm{CH}_{3}\right)_{2}$. In order to decouple electronic effects from geometrical ones, only the R1 and R2 geometries have been optimized at the B3LYP/6-31G(d,p) level of theory, keeping the PATFIA core in its experimental geometry. 
In the following, all results have been obtained with tight convergence thresholds: $10^{-8}$ a.u.for energy and $10^{-5}$ a.u.for one electron energy change. In Table 2, only hydrogen and methyl substitutions have been considered. Test calculations have also been performed with (i) hydrogen in place of the methyl group in $\mathrm{R} 1$, re-optimizing the $\mathrm{O}-\mathrm{H}$ bond length, (ii) methyl in place of hydrogen in $\mathrm{R} 1$, re-optimizing the $\mathrm{O}-\mathrm{C}$ bond length (and the methyl internal coordinates). Original PATFIA corresponds to $\mathrm{R}_{1}=\mathrm{CH}_{3}$ and $\mathrm{R}_{2}=\mathrm{H}$.

Table 2: $J$ decomposition (in $\mathrm{cm}^{-1}$ ) for PATFIA-like models, with R1 and R2 as hydrogen or methyl. $-\mathrm{H}^{*}$ means that hydrogen is positioned along the original methoxo $\mathrm{O}-\mathrm{C}$ bond (in-plane), while $-\mathrm{CH}_{3}{ }^{*}$ means that methyl is positioned along the original hydroxo $\mathrm{O}-\mathrm{H}$ bond (out-of-plane).

\begin{tabular}{rcccccc}
\hline $\mathrm{R} 1$ & $-\mathrm{CH}_{3}$ & $-\mathrm{CH}_{3}$ & $-\mathrm{H}$ & $-\mathrm{H}$ & $-\mathrm{H}^{*}$ & $-\mathrm{CH}_{3}{ }^{*}$ \\
$\mathrm{R} 2$ & $-\mathrm{CH}_{3}$ & $-\mathrm{H}$ & $-\mathrm{H}$ & $-\mathrm{CH}_{3}$ & $-\mathrm{CH}_{3}$ & $-\mathrm{CH}_{3}$ \\
\hline$J_{0}$ & 123 & 125 & 93 & 91 & 95 & 127 \\
$\Delta J_{k e}$ & -154 & -146 & -71 & -75 & -121 & -111 \\
$\Delta J_{c p}$ & -1 & 0 & 3 & 3 & -4 & 9 \\
$J_{0}+\Delta J_{k e}+\Delta J_{c p}$ & -32 & -21 & 25 & 19 & -30 & 25 \\
\hline
\end{tabular}

As previously found, ${ }^{28} \mathrm{R} 1$ is the main responsible for the magnetic character of these PATFIA-like models. Indeed, when R1 is hydrogen, the $J$ value is consistent with PATFOG one, even if the PATFIA geometry is kept. The direct exchange $J_{0}$ and the kinetic exchange $\Delta J_{k e}$ are also governed by R1 only, being reduced when hydrogen replaces methyl. However, the kinetic exchange contribution is more intense than the direct exchange when $\mathrm{R} 1$ is a methyl group, while this is the contrary when R1 is a hydrogen atom. Polarization of the non-magnetic electrons $\left(\Delta J_{c p}\right)$ remains small. Regarding the nature of R2, hydrogen or methyl, it looks like all the contributions to $J$ are rather insensitive to it. The last two columns in Table 2 show two interesting features. The magnitude of $J_{0}$ depends mainly on the chemical nature of $\mathrm{R} 1$, not on its position. Conversely, the R1 position/orientation affects $\Delta J_{k e}$.

In order to maximize the R1 and R2 substitution effects, we now report $J$ decomposition results when either $\mathrm{R} 1$ or $\mathrm{R} 2$ is a methyl group, while the other bridge is complemented with electro-donating or electro-withdrawing chemical groups. We first consider $\mathrm{R} 2=\mathrm{CH}_{3}$ 
and some carbon-centered substituents for R1 (Table 3). $\Delta J_{c p}$ is predicted to be negligible. Table 3: $J$ decomposition (in $\mathrm{cm}^{-1}$ ) for PATFIA-like models, with $\mathrm{R} 2=\mathrm{CH}_{3}$ and various R1 groups.

\begin{tabular}{rcccc}
\hline $\mathrm{R} 1$ & $-\mathrm{CF}_{3}$ & $-\mathrm{C}\left(\mathrm{OCH}_{3}\right)_{3}$ & $-\mathrm{C}\left(\mathrm{CH}_{3}\right)_{3}$ & $-\mathrm{CH}_{3}$ \\
\hline$J_{0}$ & 58 & 91 & 135 & 123 \\
$\Delta J_{k e}$ & -37 & -99 & -148 & -154 \\
$\Delta J_{c p}$ & -1 & 0 & 4 & -1 \\
$J_{0}+\Delta J_{k e}+\Delta J_{c p}$ & 20 & -8 & -9 & -32 \\
\hline
\end{tabular}

Three different kinds of R1 effects can be highlighted.

- Methyl and tertiobutyl: similar direct and kinetic exchange contributions, the latter larger than the former, resulting in an overall anti-ferromagnetic coupling.

- Trimethylformate: reduced direct and kinetic exchange contributions, the latter being still larger than the former, the total magnetic exchange remaining anti-ferromagnetic.

- Trifluoromethyl: even more reduced direct and kinetic exchange contributions, the latter smaller than the former, resulting in an overall ferromagnetic coupling.

Because these R1 groups are all positioned in equivalent orientations, roughly parallel to the $\angle \mathrm{CuOCu}$ plane, we can't rely on the usual explanation that was previously used to rationalize the different magnetic couplings in PATFIA and PATFOG. ${ }^{28}$ Indeed, the chemical nature of $\mathrm{R} 1$ is responsible for the variations of all contributions to $J$.

Now we consider $\mathrm{R} 1=\mathrm{CH}_{3}$, as it is the case in PATFIA, and a more extended set of substituents as R2 (Table 4). Whatever the nature of $\mathrm{R} 2, \Delta J_{c p}$ remains negligible with Table 4: $J$ decomposition (in $\mathrm{cm}^{-1}$ ) for PATFIA-like models, with $\mathrm{R} 1=\mathrm{CH}_{3}$ and various $\mathrm{R} 2$ groups.

\begin{tabular}{rcccccc}
\hline $\mathrm{R} 2$ & $-\mathrm{CF}_{3}$ & $-\mathrm{CH}_{3}$ & $-\mathrm{H}$ & $-\mathrm{C}\left(\mathrm{CH}_{3}\right)_{3}$ & $-\mathrm{C}\left(\mathrm{NH}_{2}\right)_{3}$ & $-\mathrm{P}\left(\mathrm{CH}_{3}\right)_{2}$ \\
\hline$J_{0}$ & 125 & 123 & 125 & 124 & 132 & 144 \\
$\Delta J_{k e}$ & -173 & -154 & -146 & -126 & -95 & -50 \\
$\Delta J_{c p}$ & -1 & -1 & -1 & -2 & -7 & -6 \\
$J_{0}+\Delta J_{k e}+\Delta J_{c p}$ & -49 & -32 & -22 & -4 & 30 & 88 \\
\hline
\end{tabular}


respect to the other contributions, while the direct exchange contribution is almost constant $\left(124 \leq J_{0} \leq 132 \mathrm{~cm}^{-1}\right)$ for the carbon-centered R2 (or H). In the case of the phosphine, this contribution slightly increases to $144 \mathrm{~cm}^{-1}$. However, the chemical nature of R2 dramatically impacts on the kinetic exchange contribution. Always antiferromagnetic, it decreases (in absolute value) from $173 \mathrm{~cm}^{-1}\left(\mathrm{R} 2=\mathrm{CF}_{3}\right)$ to $50 \mathrm{~cm}^{-1}\left(\mathrm{R} 2=\mathrm{P}\left(\mathrm{CH}_{3}\right)_{2}\right)$. In two cases $(\mathrm{R} 2$ $=\mathrm{P}\left(\mathrm{NH}_{2}\right)_{3}$ and $\left.\mathrm{P}\left(\mathrm{CH}_{3}\right)_{2}\right)$, the PATFIA-like molecule turns out to be ferromagnetic instead of anti-ferromagnetic! This striking result, and, more generally, the chemical tuning of $J$ contributions, deserve further analysis in the next section.

\section{Analysis and discussion}

As evidenced in Table 2, the magnetic character of PATFIA-like systems is mainly determined by the nature and the position of R1: anti-ferromagnetic when the bridge is methoxo, while ferromagnetic when it is hydroxo. In the original work by Lopez, ${ }^{28}$ but already evidenced earlier, ${ }^{42}$ this observation was explained as resulting from the different orientation of $\mathrm{R} 1$, more precisely to its tilt angle with respect to the $\mathrm{CuOC} u$ plane. If $\mathrm{R} 1$ is close to this plane, the compound is anti-ferromagnetic. If the out-of-plane angle is larger than $35^{\circ}$, it is ferromagnetic. In our calculations, the same change of magnetic character is due to a larger decrease of the kinetic exchange contribution (about 50\%) with respect to the decrease of the direct exchange contribution (about 25\%), when going from methoxo to hydroxo. On the other hand, substituting methyl (resp. hydrogen) with hydrogen (resp. methyl) in R1, keeping the same original orientation, indicates that the direct exchange does only depend on the nature of the R1 group. However, the kinetic exchange does depend on both the orientation and nature of R1.

Both contributions can be rationalized by tiny but sizable variations of atomic spin populations (Table 5).

Direct exchange $\left(J_{0}\right)$ ferromagnetic contribution is an intrinsic property of the magnetic 
Table 5: Mulliken atomic spin populations on selected atoms, at various stages of the $J$ decomposition procedure. If $\mathrm{R} 1$ is methyl, then the total $\mathrm{CH}_{3}$ group spin population is reported. Values in italics correspond to non-optimized positions of R1 (out-of-plane $\mathrm{CH}_{3}$, in-plane H). Atom labels are given in Figure 2.

\begin{tabular}{|c|c|c|c|c|c|c|}
\hline & $\mathrm{Cu}^{1}$ & $\mathrm{Cu}^{2}$ & $\mathrm{O}^{1}$ & $\mathrm{O}^{21}$ & $\mathrm{O}^{22}$ & $\mathrm{R} 1$ \\
\hline \multicolumn{7}{|c|}{$\mathrm{R} 1=\mathrm{CH}_{3}$} \\
\hline \multirow[t]{2}{*}{$T, R O$} & 0.634 & 0.638 & 0.132 & 0.064 & 0.073 & 0.012 \\
\hline & 0.621 & 0.626 & 0.168 & 0.063 & 0.072 & 0.028 \\
\hline \multirow[t]{2}{*}{$B S, R O$} & 0.624 & -0.627 & 0.005 & 0.062 & -0.070 & -0.000 \\
\hline & 0.611 & -0.616 & 0.006 & 0.060 & -0.069 & 0.002 \\
\hline \multirow[t]{2}{*}{$B S, U F C$} & 0.620 & -0.624 & 0.005 & 0.062 & -0.070 & -0.000 \\
\hline & 0.609 & -0.614 & 0.006 & 0.060 & -0.068 & 0.002 \\
\hline \multicolumn{7}{|c|}{$\mathrm{R} 1=\mathrm{H}$} \\
\hline \multirow[t]{2}{*}{$T, R O$} & 0.638 & 0.640 & 0.138 & 0.065 & 0.075 & 0.006 \\
\hline & 0.644 & 0.647 & 0.118 & 0.064 & 0.073 & 0.010 \\
\hline \multirow[t]{2}{*}{$B S, R O$} & 0.619 & -0.621 & 0.004 & 0.063 & -0.071 & 0.000 \\
\hline & 0.628 & -0.631 & 0.004 & 0.062 & -0.070 & 0.000 \\
\hline \multirow[t]{2}{*}{$B S, U F C$} & 0.617 & -0.620 & 0.004 & 0.063 & -0.071 & 0.000 \\
\hline & 0.625 & -0.628 & 0.004 & 0.062 & -0.070 & 0.000 \\
\hline
\end{tabular}

orbitals as obtained in the $T, R O$ calculation. The more localized they are, the less they interact. Hence the corresponding copper atomic spin populations in the T,RO solution can be used to rationalize the variations of $J_{0}$. When the methyl position is modified from in-plane to out-of-plane, there is a slight redistribution of spin population from the copper atoms to the methoxo ones. Conversely, when the hydrogen position is modified from out-ofplane to in-plane, the spin population redistribution is directed from hydroxo to the copper atoms. When methyl is replaced by hydrogen, keeping the same in-plane orientation, the spin population in copper atoms flows from R1. Conversely, when hydrogen is replaced by methyl, keeping the same out-of-plane orientation, the spin population in copper atoms flows towards R1.

Regarding the kinetic exchange contribution, ie the delocalization of a magnetic orbital towards the other magnetic site, copper spin populations are the most affected. This change is twice larger in the case of $\mathrm{R} 1=\mathrm{CH}_{3}$ than in $\mathrm{R} 1=\mathrm{H}$. This effect translates to a larger $\Delta J_{k e}$ contribution when $\mathrm{R} 1$ is methoxo. 
Overall, the methoxo vs hydroxo effect remains small from the electronic point of view. For this reason, we now turn to the analysis of spin populations in PATFIA-like systems, in which several substitutions in R1 or R2 positions have been considered. Table 3 has shown that both the direct and kinetic exchange contributions are strongly dependent of the nature of the R1 substituent. If methyl and trifluoromethyl are compared, $J_{0}$ is divided by two while $\Delta J_{k e}$ is reduced by a factor 4 . These variations can be understood by inspection of Figure 3 , in which iso-contours of the spin density are reported. Regarding the direct exchange
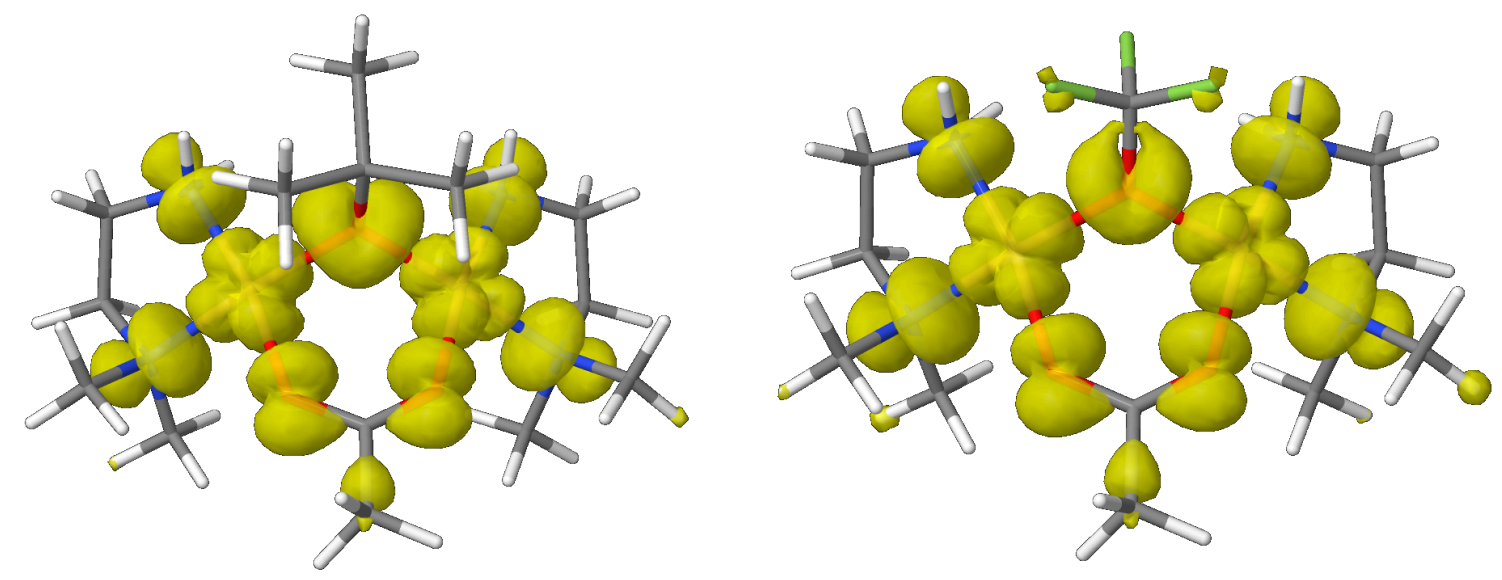

(a) T,RO (isovalue $=0.0015$ a.u.)
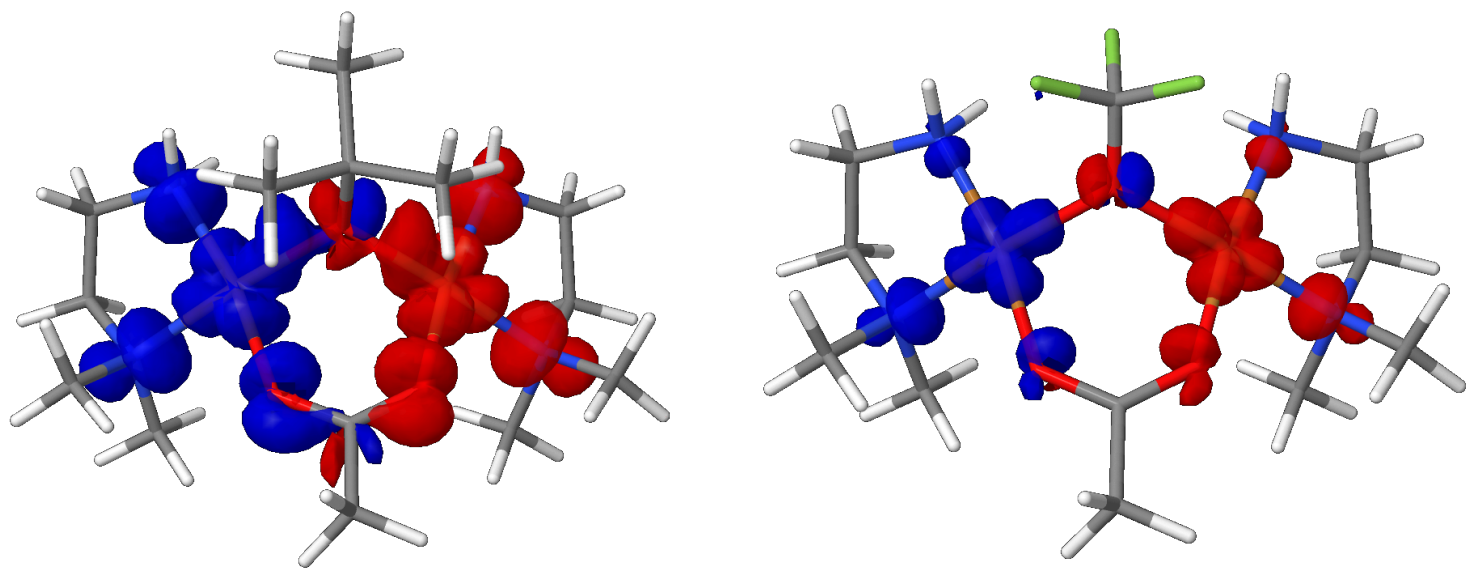

(b) BS,UFC-BS,RO (isovalue $=10^{-5}$ a.u.)

Figure 3: Spin densities (a) and spin density variations (b) for two PATFIA-like compounds, with either $\mathrm{C}\left(\mathrm{CH}_{3}\right)_{3}$ (left) or $\mathrm{CF}_{3}$ (right) as $\mathrm{R} 1$ substituent.

contribution, Figure $3 \mathrm{a}$ indicates that spin density is more delocalized when $\mathrm{R} 1=\mathrm{CF}_{3}$, 
the fluorine centers carrying a noticeable population. In other words, the F electronegative centers attract electron density outside from the bridge $\mathrm{Cu}-\mathrm{O}-\mathrm{Cu}$, which in turns lowers $J_{0}$. About the kinetic exchange contribution, the comparison of the two pictures in Figure $3 \mathrm{~b}$ clearly shows that the spin density variation is reduced when $\mathrm{R} 1=\mathrm{CF}_{3}$, in agreement with a smaller $\Delta J_{k e}$ value. In both compounds, this variation is restricted to the copper centers and the atoms directly connected to them. Accordingly, the spin density attracted by the fluorine atoms is not modified by the kinetic exchange contribution.

Table 4 has shown that the kinetic exchange is the only contribution which depends on the $\mathrm{R} 2$ substituent. Its intensity is divided by more than 3 when going from $\mathrm{R} 2=\mathrm{CF}_{3}$ to $\mathrm{P}\left(\mathrm{CH}_{3}\right)_{2}$. In Figure (4), we have reported the variations of the spin density between the $\mathrm{BS}, \mathrm{RO}$ and the BS,UFC solutions. In all cases, the spin density redistribution includes $\mathrm{Cu}$,
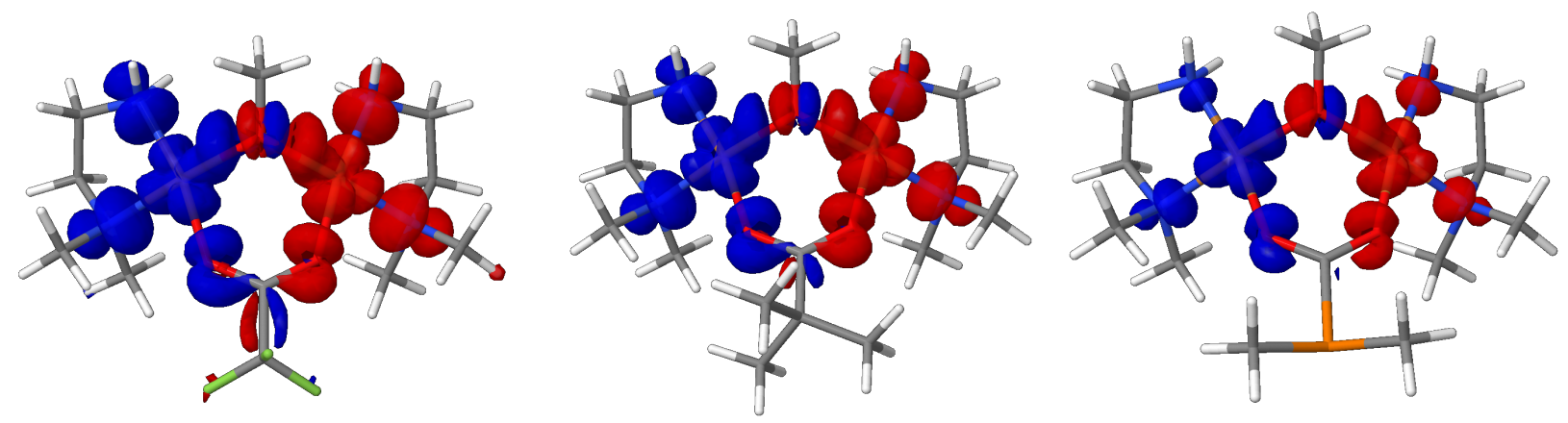

Figure 4: Spin density (isovalue $=10^{-5}$ a.u.) variations (BS,UFC-BS,RO) for three PATFIAlike compounds, with $\mathrm{CF}_{3}, \mathrm{C}\left(\mathrm{CH}_{3}\right)_{3}$ or $\mathrm{P}\left(\mathrm{CH}_{3}\right)_{2}$ as $\mathrm{R} 2$ substituent.

as well as directly-bonded $\mathrm{N}$ and $\mathrm{O}$ centers. If $\mathrm{R} 2=\mathrm{P}\left(\mathrm{CH}_{3}\right)_{2}$, there is no other variation outside these centers. If $\mathrm{R} 2=\mathrm{C}\left(\mathrm{CH}_{3}\right)_{3}$, the spin density also changes at the carboxylato $\mathrm{C}$ center. Finally, if $\mathrm{R} 2=\mathrm{CF}_{3}$, the spin density redistribution expands to the $\mathrm{C}-\mathrm{C}$ bond. These differences are perfectly in line with the reported $\Delta J_{k e}$ changes.

In closing this section we note that the particular effect of trifluoromethyl on the kinetic exchange should be highlighted. When placed at the R1 position, it causes the smallest $\Delta J_{k e}$ among all the considered substituents. Conversely, when placed at the R2 position, it causes the largest $\Delta J_{k e}$ contribution. In other words, $\mathrm{CF}_{3}$ electron-rich moiety in $\mathrm{R} 1$ 
drastically reduces the magnetic orbital delocalization, while it enhances it when $\mathrm{CF}_{3}$ is in R2. In principle, this huge effect could be understood by comparing the Hubbard $t$ (hopping energy) and $U$ (on-site minus different-sites energy difference) values. These parameters are strictly defined in the case of centro-symmetric compounds. ${ }^{41}$ However, the PATFIA-like models being close to symmetric, we have calculated approximate $t$ and $U$ values and checked their relevance by calculating a new value of the kinetic exchange contribution, $\Delta J_{k e}^{C I}$ :

$$
\Delta J_{k e}^{C I}=\frac{U-\sqrt{U^{2}+16 t^{2}}}{4}
$$

Perfect agreement between both kinetic exchange contributions fully justifies the relevance Table 6: Kinetic exchange contributions and Hubbard parameters $\left(\mathrm{in}^{-1}\right.$ ) for $\mathrm{CF}_{3}$ in $\mathrm{R} 1$ or $\mathrm{R} 2$ position. For comparison, the last line reports the same values for $\mathrm{CH}_{3}$ in both positions.

\begin{tabular}{r|c|cc}
\hline & $\Delta J_{k e}-\Delta J_{k e}^{C I}$ & $U$ & $t$ \\
\hline $\mathrm{R} 1$ & $5.10^{-3}$ & 31074 & -756 \\
$\mathrm{R} 2$ & $3.10^{-3}$ & 31077 & -1651 \\
$\mathrm{CH}_{3}$ & $3.10^{-3}$ & 31035 & -1553 \\
\hline
\end{tabular}

of the $t$ and $U$ parameters. Whatever the $\mathrm{CF}_{3}$ position, the $U$ parameters keeps the same value, implying that the energy of the ionic configurations in which both magnetic electrons are on the same $\mathrm{Cu}$ center are not affected by the position of the substituent. Conversely, the hopping energy $t$ increases by a factor of two between R1 and R2, explaining the corresponding increase of $\Delta J_{k e}$. If compared to the unsubstituted PATFIA model in which $\mathrm{CH}_{3}$ occupies both the R1 and R2 positions, it appears that $t$ is mostly affected, and reduced, when $\mathrm{CF}_{3}$ is in $\mathrm{R} 1$ position. In other words, the presence of this electron-rich substituent at position $\mathrm{R} 1$ decreases a lot the probability to transfer one magnetic electron from one $\mathrm{Cu}$ center to the other one, while it slightly increases it when placed at position R2. 


\section{Conclusions}

In this article, we have presented how the new implementation in Orca of the magnetic exchange coupling decomposition, based on broken-symmetry Kohn-Sham DFT calculations, opens the way to the computer-assisted design of substituted dinuclear non-centrosymetric compounds with either a ferro- or an anti-ferromagnetic character.

In details, we have confirmed that reduced models of PATFIA and PATFOG related systems are characterized by magnetic contributions qualitatively similar to the ones obtained with the experimental crystallographic structure. In agreement with the observation already made by López and coworkers, ${ }^{28}$ the orientation of the hydroxo vs methoxo substituents explains the different magnetic characters of PATFIA and PATFOG. However, the decomposition has allowed us to refine this interpretation by pointing out the importance of the chemical nature of these substituents, especially in the case of the direct exchange contribution.

By means of selective chemical substitutions on each bridge, we have designed PATFIAlike models which, if one can experimentally synthesize them, would exhibit either a ferromagnetic or an anti-ferromagnetic character by tuning one or several contributions to the magnetic exchange coupling. Last, we have rationalized the effect of one particular substituent, trifluoromethyle, which has been shown to drastically reduce the kinetic exchange contribution in one particular position.

\section{Acknowledgement}

GD and NF are grateful to Nathalie Guihéry and Jean-Paul Malrieu (Université Paul Sabatier, Toulouse 3, France) for all the interesting discussions and the method development over the last 10 years. GD thanks Deutscher Akademischer Austauschdienst for generous Marseille-Mulheim travel grant in 2017. Mésocentre of Aix-Marseille Université is acknowledged for allocated HPC resources. 


\section{Supporting Information Available}

Influence of ferrocene and solvent molecules on $J$ decomposition. Effect of the basis set quality and influence of relativistic effects. Spin densities of some of the compounds. Coordinates of all the compounds.

\section{References}

(1) Kahn, O. Molecular magnetism; Wiley Online Library, 1994; Vol. 25.

(2) Gatteschi, D.; Sessoli, R.; Villain, J. Molecular Nanomagnets; Oxford University Press, 2006; DOI: 10.1093/acprof : oso/9780198567530.001.0001.

(3) Pedersen, K. S.; Bendix, J.; Clérac, R. Single-molecule magnet engineering: buildingblock approaches. Chem. Commun. 2014, 50, 4396-4415, DOI: 10.1039/c4cc00339j.

(4) Demir, S.; Jeon, I.-R.; Long, J. R.; Harris, T. D. Radical ligand-containing single-molecule magnets. Coord. Chem. Rev. 2015, 289-290, 149-176, DOI: $10.1016 / j \cdot \operatorname{ccr} .2014 \cdot 10 \cdot 012$.

(5) Hay, P. J.; Thibeault, J. C.; Hoffmann, R. Orbital Interactions in Metal Dimer Complexes. J. Am. Chem. Soc. 1975, 97, 4884-4899, DOI: 10.1021/ja00850a018.

(6) Noodleman, L. Valence bond description of antiferromagnetic coupling in transition metal dimers. J. Chem. Phys. 1981, 74, 5737-5743, DOI: 10.1063/1.440939.

(7) Caballol, R.; Castell, O.; Illas, F.; de P. R. Moreira, I.; Malrieu, J. P. Remarks on the Proper Use of the Broken Symmetry Approach to Magnetic Coupling. J. Phys. Chem. A 1997, 101, 7860-7866, DOI: 10.1021/jp9711757.

(8) Barone, V.; di Matteo, A.; Mele, F.; de P. R. Moreira, I.; Illas, F. Through-bond and through-space effects in the magnetic properties of nitroxide biradicals by an integrated 
QM/MM approach including solvent effects. Chem. Phys. Lett. 1999, 302, 240-248, DOI: $10.1016 /$ S0009-2614(99)00084-6.

(9) Ruiz, E.; de Graaf, C.; Alemany, P.; Alvarez, S. Further Theoretical Evidence for the Exceptionally Strong Ferromagnetic Coupling in Oxo-Bridged $\mathrm{Cu}(\mathrm{II})$ Dinuclear Complexes. The Journal of Physical Chemistry A 2002, 106, 4938-4941, DOI: 10.1021/jp015565b.

(10) Ciofini, I.; Daul, C. A. DFT calculations of molecular magnetic properties of coordination compounds. Coordination Chemistry Reviews 2003, 238-239, 187-209, DOI: 10.1016/S0010-8545(02)00330-2.

(11) Illas, F.; de P. R. Moreira, I.; Bofill, J. M.; Filatov, M. Extent and limitations of densityfunctional theory in describing magnetic systems. Phys. Rev. B 2004, 70, 132414, DOI: 10.1103/PhysRevB.70.132414.

(12) Moreira, I. d. P. R.; Illas, F. A unified view of the theoretical description of magnetic coupling in molecular chemistry and solid state physics. Phys. Chem. Chem. Phys. 2006, 8, 1645-1659, DOI: 10.1039/B515732C.

(13) Queralt, N.; Taratiel, D.; de Graaf, C.; Caballol, R.; Cimiraglia, R.; Angeli, C. On the applicability of multireference second-order perturbation theory to study weak magnetic coupling in molecular complexes. Journal of Computational Chemistry 2008, 29, 9941003, DOI: $10.1002 /$ jcc. 20859.

(14) Vancoillie, S.; Rulisek, L.; Neese, F.; Pierloot, K. Theoretical Description of the Structure and Magnetic Properties of Nitroxide- $\mathrm{Cu}(\mathrm{II})$-Nitroxide Spin Triads by Means of Multiconfigurational Ab Initio Calculations. J. Phys. Chem. A 2009, 113, 6149-6157, DOI: $10.1021 / j p 900822 v$, PMID: 19413285.

(15) Neese, F. Prediction of molecular properties and molecular spectroscopy with density 
functional theory: From fundamental theory to exchange-coupling. Coord. Chem. Rev. 2009, 253, 526-563, DOI: 10.1016/j.ccr.2008.05.014.

(16) Phillips, J. J.; Peralta, J. E. Magnetic Exchange Couplings from Semilocal Functionals Evaluated Nonself-Consistently on Hybrid Densities: Insights on Relative Importance of Exchange, Correlation, and Delocalization. J. Chem. Theory Comput. 2012, 8, 31473158, DOI: $10.1021 /$ ct3004904.

(17) Malrieu, J. P.; Caballol, R.; Calzado, C. J.; de Graaf, C.; Guihéry, N. Magnetic Interactions in Molecules and Highly Correlated Materials: Physical Content, Analytical Derivation, and Rigorous Extraction of Magnetic Hamiltonians. Chem. Rev. 2014, 114, 429 - 492, DOI: $10.1021 /$ cr300500z.

(18) de Graaf, C.; Broer, R. Magnetic Interactions in Molecules and Solids (Theoretical Chemistry and Computational Modelling); Theoretical Chemistry and Computational Modelling; Springer, 2015; DOI: 10.1007/978-3-319-22951-5.

(19) Malrieu, J.-P.; Trinquier, G. Communication: Proper use of broken-symmetry calculations in antiferromagnetic polyradicals. J. Chem. Phys. 2016, 144, 211101, DOI: $10.1063 / 1.4953040$.

(20) Roemelt, M.; Krewald, V.; Pantazis, D. A. Exchange Coupling Interactions from the Density Matrix Renormalization Group and N-Electron Valence Perturbation Theory: Application to a Biomimetic Mixed-Valence Manganese Complex. J. Chem. Theory Comput. 2017, 14, 166-179, DOI: 10.1021/acs.jctc.7b01035.

(21) Terencio, T.; Bastardis, R.; Suaud, N.; Maynau, D.; Bonvoisin, J.; Malrieu, J. P.; Calzado, C. J.; Guihéry, N. Physical analysis of the through-ligand long-distance magnetic coupling: spin-polarization versus Anderson mechanism. Phys. Chem. Chem. Phys. 2011, 13, 12314-12320, DOI: 10.1039/C1CP20179D. 
(22) Sinnecker, S.; Neese, F.; Noodleman, L.; Lubitz, W. Calculating the Electron Paramagnetic Resonance Parameters of Exchange Coupled Transition Metal Complexes Using Broken Symmetry Density Functional Theory: Application to a MnIII/MnIV Model Compound. J. Am. Chem. Soc. 2004, 126, 2613-2622.

(23) Coulaud, E.; Guihéry, N.; Malrieu, J.-P.; Hagebaum-Reignier, D.; Siri, D.; Ferré, N. Analysis of the physical contributions to magnetic couplings in broken symmetry density functional theory approach. J. Chem. Phys. 2012, 13\%, 114106, DOI: $10.1063 / 1.4752412$.

(24) Coulaud, E.; Malrieu, J.-P.; Guihéry, N.; Ferré, N. Additive Decomposition of the Physical Components of the Magnetic Coupling from Broken Symmetry Density Functional Theory Calculations. J. Chem. Theory Comput. 2013, 9, 3429 - 3436, DOI: 10.1021/ct400305h.

(25) Ferré, N.; Guihéry, N.; Malrieu, J.-P. Spin decontamination of broken-symmetry density functional theory calculations: deeper insight and new formulations. Phys. Chem. Chem. Phys. 2015, 17, 14375-14382, DOI: 10.1039/c4cp05531d.

(26) Le Guennic, B.; Ferré, N. Analysis of the Magnetic Exchange Interaction in HalideBridged $\mathrm{Cu}(\mathrm{II})$ Binuclear Complexes: Deciphering the Paths. Curr. Inorg. Chem. 2014, 3, 235-241, DOI: 10.2174/1877944103666140110225547.

(27) Neese, F. The ORCA program system. WIREs Comput. Mol. Sci. 2011, 2, 73-78, DOI: 10.1002/wcms. 81 .

(28) López, C.; Costa, R.; Illas, F.; de Graaf, C.; Turnbull, M. M.; Landee, C. P.; Espinosa, E.; Mata, I.; Molins, E. Magneto-structural correlations in binuclear copper(ii) compounds bridged by a ferrocenecarboxylato $(-1)$ and an hydroxo- or methoxo-ligands. Dalton Trans. 2005, 2322, DOI: 10.1039/b502264a. 
(29) Moreira, I. d. P. R.; Costa, R.; Filatov, M.; Illas, F. Restricted Ensemble-Referenced Kohn-Sham versus Broken Symmetry Approaches in Density Functional Theory: Magnetic Coupling in Cu Binuclear Complexes. J. Chem. Theory Comput. 2007, 3, 764-774, DOI: $10.1021 /$ ct7000057.

(30) Valero, R.; Costa, R.; de P. R. Moreira, I.; Truhlar, D. G.; Illas, F. Performance of the M06 family of exchange-correlation functionals for predicting magnetic coupling in organic and inorganic molecules. J. Chem. Phys. 2008, 128, 114103, DOI: $10.1063 / 1.2838987$.

(31) Rivero, P.; de P. R. Moreira, I.; Illas, F.; Scuseria, G. E. Reliability of range-separated hybrid functionals for describing magnetic coupling in molecular Systems. J. Chem. Phys. 2008, 129, 184110, DOI: 10.1063/1.3006419.

(32) Valero, R.; Illas, F.; Truhlar, D. G. Magnetic Coupling in Transition-Metal Binuclear Complexes by Spin-Flip Time-Dependent Density Functional Theory. J. Chem. Theory Comput. 2011, 7, 3523-3531, DOI: 10.1021/ct200393s.

(33) Zhekova, H. R.; Seth, M.; Ziegler, T. Calculation of the exchange coupling constants of copper binuclear systems based on spin-flip constricted variational density functional theory. J. Chem. Phys. 2011, 135, 184105, DOI: 10.1063/1.3658643.

(34) Zhang, Y.; Jiang, H. Intra- and Interatomic Spin Interactions by the Density Functional Theory plus U Approach: A Critical Assessment. J. Chem. Theory Comput. 2011, 7, 2795-2803, DOI: 10.1021/ct200299s.

(35) Orms, N.; Krylov, A. I. Singlet-triplet energy gaps and the degree of diradical character in binuclear copper molecular magnets characterized by spin-flip density functional theory. Phys. Chem. Chem. Phys. 2018, DOI: 10.1039/c7cp07356a.

(36) Costa, R.; Reta, D.; de P. R. Moreira, I.; Illas, F. Post-B3LYP Functionals Do Not 
Improve the Description of Magnetic Coupling in $\mathrm{Cu}(\mathrm{II})$ Dinuclear Complexes. J. Phys. Chem. A 2018, 122, 3423-3432, DOI: 10.1021/acs. jpca.7b12663.

(37) https://www.ccdc.cam.ac.uk/. https://www.ccdc.cam.ac.uk/.

(38) Pipek, J.; Mezey, P. G. A fast intrinsic localization procedure applicable for ab initio and semiempirical linear combination of atomic orbital wave functions. J. Chem. Phys. 1989, 90, 4916-4926.

(39) Foster, J. M.; Boys, S. F. Canonical Configuration Interaction Procedure. Rev. Mod. Phys. 1960, 32, 300.

(40) Assfeld, X.; Rivail, J.-L. Quantum chemical computations on parts of large molecules: the ab initio local self consistent field method. Chem. Phys. Lett. 1996, 263, 100-106.

(41) David, G.; Guihéry, N.; Ferré, N. What Are the Physical Contents of Hubbard and Heisenberg Hamiltonian Interactions Extracted from Broken Symmetry DFT Calculations in Magnetic Compounds? J. Chem. Theory Comput. 2017, 13, 6253-6265, DOI: 10.1021/acs.jctc. $7 \mathrm{~b} 00976$.

(42) Ruiz, E.; Alemany, P.; Alvarez, S.; Cano, J. Toward the Prediction of Magnetic Coupling in Molecular Systems: Hydroxo- and Alkoxo-Bridged $\mathrm{Cu}(\mathrm{II})$ Binuclear Complexes. $J$. Am. Chem. Soc. 1997, 119, 1297-1303, DOI: 10.1021/ja961199b. 


\section{Graphical TOC Entry}

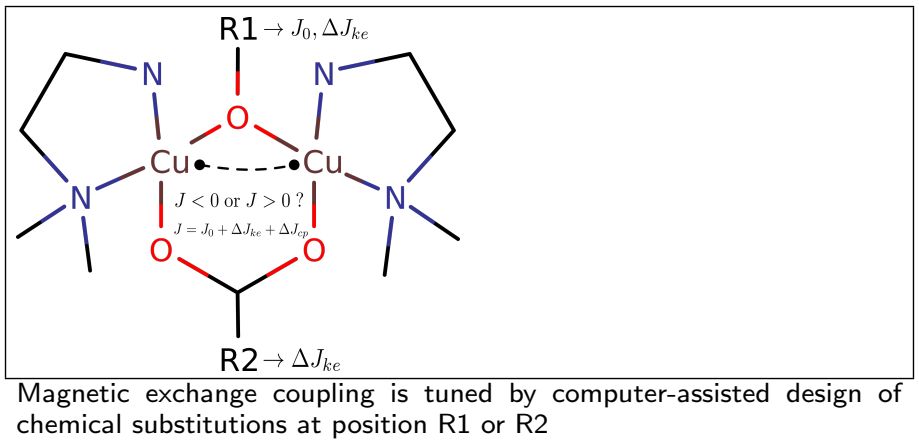

